\title{
The Predictors of Psychological Status among Primary Breast Cancer Patients in Japan
}

\author{
Takako Maeda1, Isamu Morishima², Ei Ueno², Takeshi Umemoto², \\ Kyoko Dogo Sasaki ${ }^{3}$ \\ ${ }^{1}$ Faculty of Nursing, Ibaraki Prefectural University of Health Science, Ibaraki, Japan \\ ${ }^{2}$ Department of Senology, Tsukuba Medical Center Hospital, Ibaraki, Japan \\ ${ }^{3}$ Department of Plastic and Reconstructive Surgery, Dokkyo Medical University Koshigaya Hospital, Saitama, \\ Japan \\ Email: maedat@ipu.ac.jp
}

Received 26 December 2013; revised 27 January 2014; accepted 28 February 2014

Copyright (C) 2014 by authors and Scientific Research Publishing Inc.

This work is licensed under the Creative Commons Attribution International License (CC BY).

http://creativecommons.org/licenses/by/4.0/

(c) (i) Open Access

\section{Abstract}

This study examined the psychological status of Japanese ambulatory patients with primary breast cancer, with a focus on evaluating the impact of the patients' self-repressive trait and the role of previously identified associated factors, including social support, attributes, and diseaserelated variables. The subjects included 112 women [mean (SD) age: 58.3 (12.7)] in Japan with postoperative primary breast cancer. The results showed that around $40 \%$ of the subjects were in a psychologically vulnerable situation. In addition, the self-repressive trait and support from family were significant predictors of both anxiety [self-repressive trait $(\beta=0.30, p=0.000)$ and family support $(\beta=-0.37, p=0.001)$ ] and depression [self-repressive trait $(\beta=0.26, p=0.002)$ and family support $(\beta=-0.42, p=0.000)]$, whereas attributes and disease-related variables were not. Health care providers need to recognize that self-repressive patients may seem to be well-adjusted when, in fact, they are suffering. Careful assessment of self-repression and psychological status is needed. Family support can contribute to a better psychological status of patients. Nurses need to encourage and provide support for not only the patients but also the family members so that family members can also effectively care for the patients' needs.

\section{Keywords}

Breast Cancer; Japanese; Anxiety; Depression; Self-Repressive Trait; Family Support 


\section{Introduction}

\subsection{Background}

Breast cancer is the most common cancer in Japanese women and its prevalence has been rapidly increasing [1]. It was estimated that over 60,000 Japanese women would develop breast cancer in 2007 [2].

Diagnosis, treatment and living with the breast cancer are extremely stressful. One study suggested that approximately 30\% (range, 20\% - 40\%, depending on the country where the research was conducted) of breast cancer survivors experience psychiatric distress [3]-[7].

Some researchers suggested that the self-repressive trait is closely related to physiological function and/or psychological status and that excessive repression may result in serious problems. For instance, breast cancer patients with negative emotional repression are more fatalistic, experience a state of hopelessness [7] [8], and have a deeper level of depression [6] [9] [10]. This notion has been supported by several domestic and international studies [11] [12].

The self-repressive trait is commonly observed in Japanese people. Munakata [13] defined the self-repressive trait as, the tendency to repress emotions, feelings, or one's own needs, paying too much attention to fulfilling other people's demands or expectations. The results of a social survey conducted in Japan showed a positive correlation between the self-repressive trait and psychosomatic disorders [14]. However, this association has not been studied in breast cancer patients in Japan.

Stressor for breast cancer patients may not only be limited to disease treatment or disease progression, but also may include household responsibilities, social obligations, and interpersonal relationships. Personal propensity may influence the way patients cope with these daily hassles and may lead to a psychosomatic outcome. However, these findings have rarely focused on nursing practices in Japan. In fact, some support programs, which were commonly provided by nurses, mainly included educational intervention (providing medical knowledge or symptom management) and enhancing perceived social support [15]-[17]. However, attention was not given to the patient's personal disposition. Spiegel et al. [18] suggested that there is a danger that repressive patients might appear to be well-adjusted because they try not to show their distress. Health care providers may be overlooking a patient's state of adjustment, even though the patient is suffering.

Other than the self-repressive trait, there are various predictors of the psychological state of breast cancer patients. For instance, being young [10] [19] [20], unmarried [10] [19], and having illness-related factors, including disease severity, treatment options, and health status, have also been identified as risk factors in the patients' psychological status [21]-[23]. Social support can also be an important predictor of psychological status in cancer patients [24]-[26]. Assessment of significant predictors of the psychological status in breast cancer patients may depend on which variables were evaluated.

In this study, we explored the impact of these variables, with a particular focus on the self-repressive trait, on the psychological status of Japanese ambulatory patients with primary breast cancer.

\subsection{Definition of the Self-Repressive Trait}

According to Munakata's definition [13], the self-repressive trait is defined as the tendency to repress emotions, feelings or one's own needs, and paying too much attention to fulfilling other people's demands or expectations to avoid being disliked by others. The self-repressive trait has a close relationship with interpersonal dependency, and it might derive from the Japanese culture or social norms. Self-repressors tend to have patience and rarely show their own suffering.

\subsection{Conceptual Framework}

The current study was based on Lazarus and Folkman’s stress, appraisal, and coping model [27]. We hypothesized the relationship among variables as shown in Figure 1.

The stress, appraisal, and coping model suggested that an individual's psychological symptoms are the results of relationships between the individual and an environment that is appraised by him or her as either taxing or exceeding his or her resources and endangering his or her well-being [27]. Then, through the process of appraisal, which evaluates either the availability of coping options or likelihood of dealing with the situation, it eventually affects the psychological status of the patient.

Self-repressors cope with stressful situations by repressing their emotions or their own needs to meet someone 


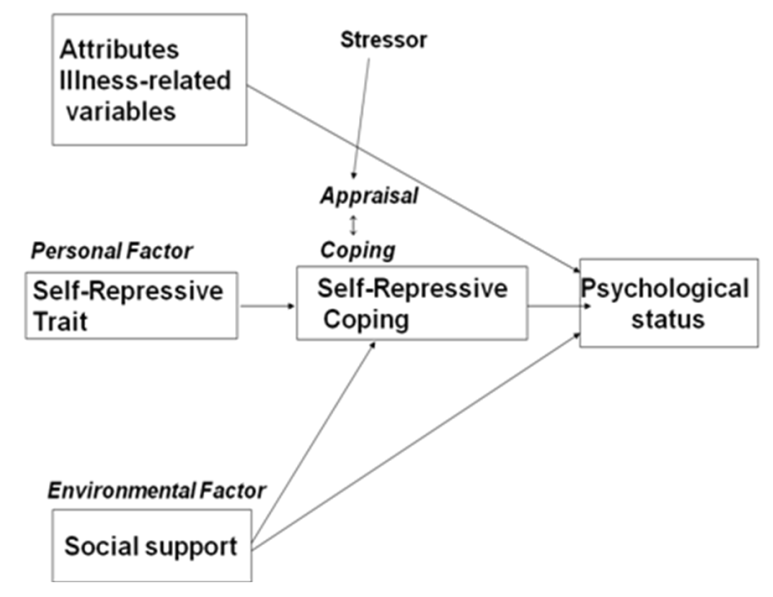

Figure 1. Conceptual framework.

else's expectations. This coping may be close to a type of emotion-focused coping rather than a problem-focused coping. A previous study suggested that there is a positive relationship between avoidance and a self-repressive trait [14]. This type of coping may not lead to resolving the stress, and then the psychological burden may be increased when the stress is prolonged [14]. We hypothesize that self-repressive coping to stressful situation in their daily life will accumulate stress, resulting in a psychological crisis. In the current study, a self-repression scale [13] was used to assess self-repressive traits by scoring the coping tendencies of patients. Psychological status was regarded as an outcome indicator in this study, and the Hospital Anxiety and Depression Scale (HADS) [28] was used to assess anxiety and depression in patients.

Social support can be considered as an environmental factor and is a powerful predictor of psychological status [24]-[26]. It helps buffer the negative consequences of both the illness and its treatment and it lessen depression in cancer patients. Since environmental factors influence the process of appraisal and coping [27], an arrow was positioned between social support and self-repressive coping in Figure 1 to indicate the relationship. The Multidimensional Scale of Perceived Social Support [29] [30] was used in this study to assess perceived social support from family, friends, and significant others.

Attributes and disease-related variables, including disease stage, surgery type, and type of therapy that the patients are receiving, may have an impact on their psychological status.

\subsection{Purpose of the Study}

The study aimed to examine the psychological status of Japanese ambulatory patients with primary breast cancer, especially focusing on the impact of the patients' self-repressive trait on their psychological status when previously identified associated factors were tested simultaneously. Previously identified associated factors include perceived social support, attributes, and disease-related variables (surgery type, stage, and type of therapy that the patients are receiving).

\subsection{Ethical Consideration}

This study was approved by the institutional ethical committees of both the authors' institution and the hospital where the survey was conducted.

\section{Methods}

\subsection{Design, Sample, and Procedure}

The study had a cross-sectional, correlational predictive design. Subjects were ambulatory postoperative breast cancer patients at the hospital where the study was conducted. Eligibility criteria for patient selection were: confirmed primary breast cancer and previous surgery; being followed-up as an ambulatory patient; not suffering from a severe mental disorder.

Questionnaires were distributed to the eligible patients after a physician's clinical examination. The patients 
received an explanation regarding the study purpose and procedure by a researcher. Data were collected through the mail. Returning the completed questionnaire indicated that the patient agreed to participate in the study.

\subsection{Study Variables and Scales}

\subsubsection{Attributes}

Age, marital status, having or not having children, and employment status.

\subsubsection{Disease-Related Variables and Treatment}

Time elapsed since hospital discharge from surgery, surgery type, disease stage, and type of therapy if they are receiving therapy.

\subsubsection{Scales}

\section{1) Self-repression}

Munakata's self-repression scale [13] is a 10-item Likert-type scale that assesses self-repressive traits by scoring the coping tendency of patients. Sample items include "I endeavor to please others," "I tend to repress my feelings," and "I try to be patient even when I am having a difficult time." The responses that approximated to self-repression were scored 2 (I always do so), 1 (I sometimes do so), or 0 (I never do so). A high score indicates an increased likelihood of self-repression. This scale has adequate validity and reliability [13], and Cronbach's $\alpha$ in this study was 0.67 .

2) Hospital Anxiety and Depression Scale (HADS)

This 14-item questionnaire was developed by Zigmond et al. [31] to assess anxiety and depression as two dimentions, which has been validated in patients with cancer [32]. Sample items on the anxiety scale include "Do you feel tense and wound up?" "Do you worry a lot?". Items on the depression scale include "Do you take as much interest in things as you used to?" "Do you feel cheerful?". Each item has a four-point ( 0 - 3) response category. Some items score using reverse scoring. Higher scores indicate a greater tendency toward anxiety and depression. Kitamura [28] translated this questionnaire into Japanese, and adequate validity and reliability have been confirmed [4]. Cronbach's $\alpha$ values in this study were 0.67 for anxiety and 0.81 for depression.

3) Multidimensional Scale of Perceived Social Support

This 12-item Likert-type scale was developed by Zimet et al. [29] to assess three-dimensional sources of social support from family, friends, and significant others. Each dimension has four items. Sample items for perceived social support include "my family really tries to help me," "I can count on my friends when things go wrong," and "I have a special person who is a real source of comfort me." The responses were scored 1 (very strongly disagree) to 7 (very strongly agree), with higher scores indicating higher recognition of social support. This scale has adequate validity and reliability [29]. This instrument has been translated into Japanese and has been confirmed for reliability and for validity [30]. Cronbach's $\alpha$ values in this study were 0.93 for support from family, 0.94 for support from friends, and 0.94 for support from significant others.

This questionnaire was a self-administered test; however, data regarding the surgery type, surgery site, recurrent status, and disease stage were obtained from medical records by the investigators.

\subsection{Data Analyses}

Four main analyses were performed using the Statistical Package for Social Sciences, version 20 (IBM Inc., Tokyo, Japan) and included descriptive analyses of patient sample characteristics. The Mann-Whitney $U$ and Kruskal-Wallis tests were used to determine whether there was a significant difference in anxiety level or depression level between subgroups of patients classified according to attribute variables or disease-related variables. Pearson's correlation coefficient was used to estimate the relationship between anxiety level or depression level and the study variables. Multiple regression analyses (forced-entry method) were used to examine the effects of the study variables on anxiety and on depression.

\section{Results}

\subsection{Patient Characteristics}

The study was conducted from February to July, 2012. Two hundred and ten questionnaires were distributed to 
eligible patients and 129 were returned. Ten of the 129 were excluded because $50 \%$ or more of the sections on the questionnaire were not completed. Seven subjects failed to identify their medical records. Therefore, the data from 112 patients (response rate, 53.0\%) were used for the analyses.

All subjects were female and their mean [standard deviation (SD)] age was 58.3 (12.7) years (range, 32 - 86 years). The demographic and clinical characteristics of this study sample are summarized in Table 1.

Table 2 shows the descriptive statistics of the study tools. The mean \pm SD anxiety and depression were $7.1 \pm$ 2.9 and $4.6 \pm 3.5$, respectively. The mean \pm SD score for self-repression was $11.3 \pm 2.9$. The mean \pm SD scores for perceived social support from family, friends, and significant others were $22.4 \pm 4.6,19.8 \pm 5.4$, and $22.0 \pm$ 5.6, respectively (Table 2). Regarding psychological status, 42 (37.5\%) patients scored above 8 points for anxiety, which was set as the cut-off point for the need for psychological help (Table 3). Similarly, 49 (43.8\%) patients scored above this cut-off point for depression (Table 3).

\subsection{Differences in Anxiety and in Depression According to Attributes and Disease-Related Variables}

Using the Mann-Whitney $U$ test and Kruskal-Wallis test, no statistical differences in levels of anxiety were found among the subgroups of subjects when categorized by patient characteristics which include attributes or disease-related variables (Table 4). Moreover, no significant differences in depression levels were found when subjects were subgrouped according to patient characteristics.

\subsection{Relationship between Social Support and Self-Repression}

No significant relation was observed between the self-repression and either support from family $(r=-0.08, p=$ $0.37)$, support from friends $(r=-0.05, p=0.58)$, or support from significant others $(r=-0.06, p=0.49)$.

\subsection{Related Variables and Determinants of Anxiety and Depression}

Pearson's product-moment correlation coefficient was calculated to examine the relationship between anxiety score and the following variables: age, self-repression, and perceived multidimensional social support. Then, a multiple regression analysis, in which anxiety was set as the dependent variable, and significantly related variables were set as independent variables, was performed. The same procedure was used but replaced the anxiety score with the depression score.

The results showed a significant relationship between anxiety and self-repression $(r=0.32, p=0.000)$, support from family $(r=-0.41, p=0.000)$, and support from significant others $(r=-0.29, p=0.001)$. No significant correlations were observed between anxiety and either age or support from friends. Multiple regression analysis, in which anxiety was set as the dependent variable and significantly related variables, such as self-repression, support from family, and support from significant others, were set as independent variables and showed that self-repression $(\beta=0.30, \mathrm{p}=0.000)$ and support from family $(\beta=-0.37, \mathrm{p}=0.001)$ had significant effects on anxiety, indicating a $24 \%$ variance.

Additionally, the same analysis method was performed for depression. The results showed a significant relationship between depression and self-repression $(r=0.30, p=0.001)$, support from family $(r=-0.47, p=0.000)$, support from friends $(r=-0.27, p=0.004)$, and support from significant others $(r=-0.32, p=0.000)$. No significant correlation was observed between depression and age. A multiple regression analysis was performed using depression as the dependent variable, and these significantly related variables were used as independent variables. Consequently, self-repression $(\beta=0.26, \mathrm{p}=0.002)$, and support from family $(\beta=-0.42, \mathrm{p}=0.000)$ were found to be significant predictors of depression, indicating a $27 \%$ variance (Table 5).

\section{Discussion}

We examined the psychological status and its determinants, especially focusing on the self-repressive trait in ambulatory Japanese breast cancer patients based on a devised conceptual framework (Figure 1). Among these set variables, self-repression and support from family were significant predictors of anxiety and depression. The psychological status of the patients and their relation to all the tested variables are discussed below.

The mean age of the patients was 58.3 years, with most patients being in their 40s - 60s. Approximately $38 \%$ of the subjects scored higher than the cut-off point for anxiety, and approximately $43 \%$ of the subjects scored 
Table 1. Patient demographic, medical, and physical data $(\mathrm{n}=112)$.

\begin{tabular}{|c|c|}
\hline \multicolumn{2}{|l|}{ Age } \\
\hline Mean (SD) & $58.3(12.7)$ \\
\hline \multirow[t]{2}{*}{ Minimum/maximum } & $32 / 86$ \\
\hline & Number (\%) \\
\hline \multicolumn{2}{|l|}{ Time elapsed since discharge } \\
\hline Less than 1 year & $49(43.8)$ \\
\hline 1 year - 3 year & $27(24.1)$ \\
\hline 3 year - 5 year & $12(10.7)$ \\
\hline 5 year - 10 year & $20(17.9)$ \\
\hline Over 10 year & $4(3.6)$ \\
\hline \multicolumn{2}{|l|}{ Marital status } \\
\hline Married & $81(72.3)$ \\
\hline Unmarried/divorced/widowed & $30(26.8)$ \\
\hline Missing & $1(0.9)$ \\
\hline \multicolumn{2}{|l|}{ Children } \\
\hline Having children & $95(84.8)$ \\
\hline Not having children & $16(14.3)$ \\
\hline Missing data & $1(0.9)$ \\
\hline \multicolumn{2}{|l|}{ Employment status } \\
\hline Employed & $56(50.0)$ \\
\hline Unemployed & $55(49.1)$ \\
\hline Missing data & $1(0.9)$ \\
\hline \multicolumn{2}{|l|}{ Type of surgery } \\
\hline Mastectomy & $39(34.8)$ \\
\hline Lumpectomy & $73(65.2)$ \\
\hline \multicolumn{2}{|l|}{ Type of therapy ${ }^{\mathrm{a}}$} \\
\hline Chemotherapy & $10(8.9)$ \\
\hline Radiation therapy & $7(6.3)$ \\
\hline Hormonal therapy & $55(49.1)$ \\
\hline Molecular-targeted therapy & $9(8.0)$ \\
\hline Check-up only & $26(23.2)$ \\
\hline \multicolumn{2}{|l|}{ Disease stage $^{\mathrm{a}}$} \\
\hline 0 & $6(5.4)$ \\
\hline I & $56(50.0)$ \\
\hline II & $39(34.8)$ \\
\hline III & $11(9.8)$ \\
\hline
\end{tabular}

Table 2. Mean and standard deviation of the study tools.

\begin{tabular}{cccc}
\hline Variables & $\begin{array}{c}\text { Possible } \\
\text { score range }\end{array}$ & Mean \pm SD & $\begin{array}{c}\text { Actual score } \\
\text { range }\end{array}$ \\
\hline HADS (anxiety) & $0-21$ & $7.1 \pm 2.9$ & $2-16$ \\
HADS (depression) & $0-21$ & $4.6 \pm 3.5$ & $0-17$ \\
Self-repression & $0-20$ & $11.3 \pm 2.9$ & $3-18$ \\
Support (family) & $4-28$ & $22.4 \pm 4.6$ & $4-28$ \\
Support (friends) & $4-28$ & $19.8 \pm 5.4$ & $4-28$ \\
Support (significant other) & $4-28$ & $22.0 \pm 5.6$ & $4-28$ \\
\hline
\end{tabular}

Abbreviation: HADS, Hospital Anxiety and Depression Scale. 
Table 3. Psychological morbidity of the subjects $(n=112)$.

\begin{tabular}{ccc}
\hline Scale (Cut-off point) & Score & Number of subjects (\%) \\
\hline HADS (anxiety) (7/8) & 7 or lower & $69(61.6)$ \\
& 8 or higher & $42(37.5)$ \\
HADS (depression) (4/5) & 4 or lower & $62(55.4)$ \\
& 5 or higher & $49(43.8)$ \\
\hline
\end{tabular}

Abbreviation: HADS, Hospital Anxiety and Depression Scale. ${ }^{\text {} H i g h e r ~ s c o r e ~ i n d i c a t e s ~ a ~ p s y c h o l o g i c a l l y ~ v u l n e r a b l e ~ s t a t e . ~}$

Table 4. Differences in anxiety and in depression related to attributes and disease-related variables $(\mathrm{n}=112)$.

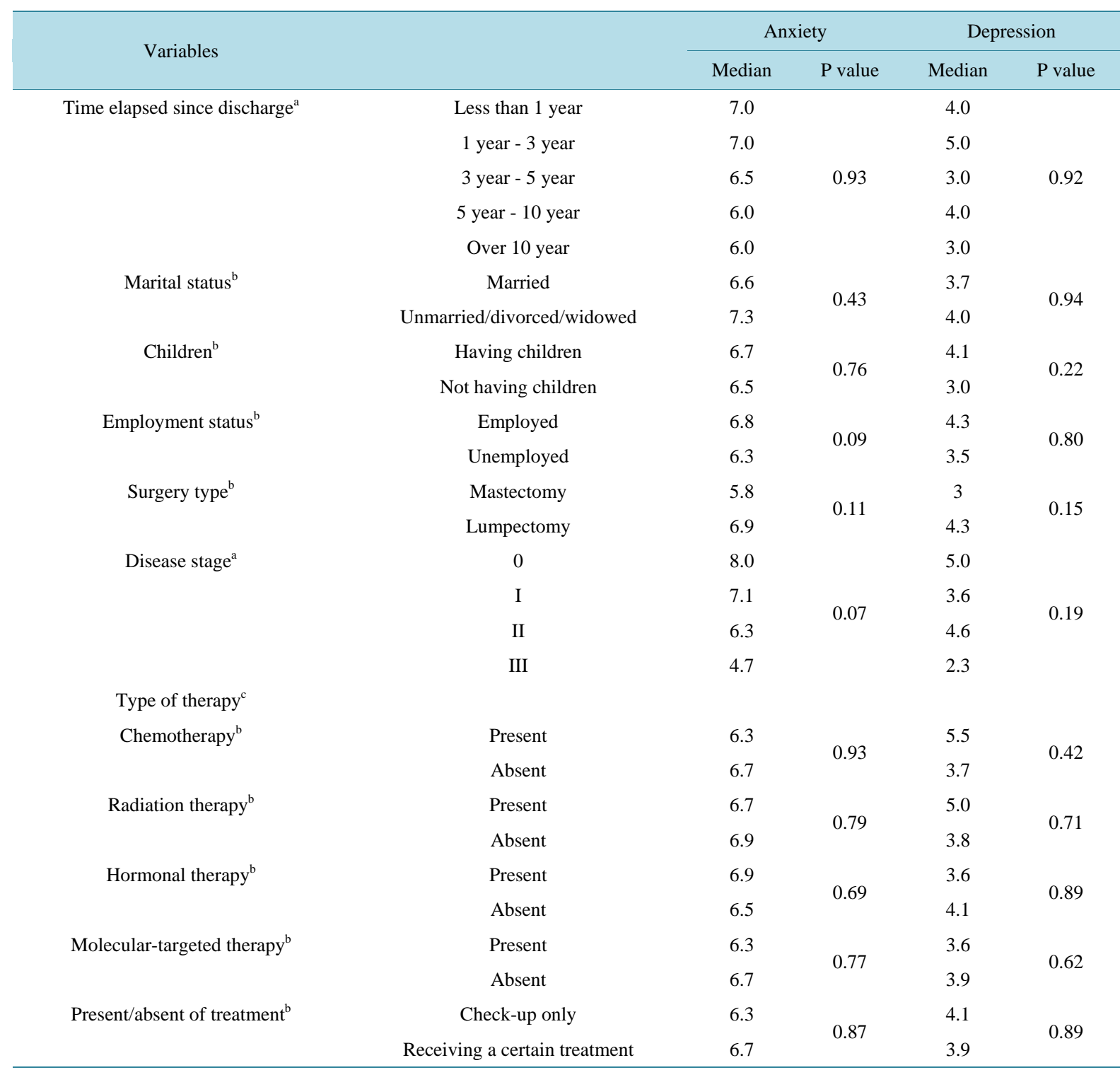

${ }^{\mathrm{a}}$ Kruskal-Wallis test. ${ }^{\mathrm{b}} \mathrm{Mann}-$ Whitney's U test. ${ }^{\mathrm{c}}$ Type of therapy that the patients were receiving at the time of the study.

higher than the cut-off point for depression, indicating that around $40 \%$ of the subjects were psychologically vulnerable and may require support.

There were no significant differences in either anxiety or depression level between subgroups of patients classified according to attribute variables, such as age, marital status, having or not having children, and employ- 
Table 5. Related variables and predictors of anxiety and depression based on Pearson's product-moment correlation coefficient and multiple regression analyses (forced-entry method) $(\mathrm{n}=112)$.

\begin{tabular}{|c|c|c|c|c|}
\hline \multicolumn{5}{|c|}{ Dependent variable: anxiety } \\
\hline Independent variable & r & $\mathrm{p}$ value & $\beta$ & $\mathrm{p}$ value \\
\hline Age $^{a}$ & -0.67 & 0.47 & & \\
\hline Self-repression & 0.32 & 0.000 & 0.30 & 0.000 \\
\hline Support (family) & -0.41 & 0.000 & -0.37 & 0.001 \\
\hline Support (friends) ${ }^{a}$ & -0.14 & 0.13 & & \\
\hline Support (significant other) & -0.29 & 0.001 & & \\
\hline Adjusted $\mathrm{R}^{2}$ & & & 0.24 & 0.000 \\
\hline $\mathrm{F} / \mathrm{df}$ & & & $13.0 / 3$ & \\
\hline \multicolumn{5}{|c|}{ Dependent variable: depression } \\
\hline Independent variable & r & $\mathrm{p}$ value & $\beta$ & $\mathrm{p}$ value \\
\hline $\mathrm{Age}^{\mathrm{a}}$ & 0.21 & 0.82 & & \\
\hline Self-repression & 0.30 & 0.001 & 0.26 & 0.002 \\
\hline Support (family) & -0.47 & 0.000 & -0.42 & 0.000 \\
\hline Support (friends) & -0.27 & 0.004 & & \\
\hline Support (significant other) & -0.32 & 0.000 & & \\
\hline Adjusted $\mathrm{R}^{2}$ & & & 0.27 & 0.000 \\
\hline F/df & & & $11.4 / 4$ & \\
\hline
\end{tabular}

${ }^{a}$ Only a correlation analysis was performed; the variable was not independent of either anxiety or depression.

ment status, and to disease-related variables, such as time elapsed since discharge, differences in surgery type, disease stage, and type of therapies that the patients were receiving at the time of the study. These findings agreed with some but did not agree with all previous studies [10] [19]-[23]. For instance, Andreu et al. [6] suggested there was no relation between distress and demographic and medical factors, however, psychosocial aspects were significant risk factors for women with breast cancer. In addition, Bardwell et al. [10] reported before entry of psychosocial variables for the binary logistic regression analysis, some attribute variables and physical functioning were significant risk factors for depressive symptoms, but after inclusion of psychosocial variables, only psychosocial variables were significant risk factors. These results, including the current study, suggested the need to pay closer attention to psychosocial aspects of breast cancer patients.

Regarding the self-repressive trait, the mean self-repression score was $11.3 \pm 2.9$. A previous study estimated self-repression in 32 Japanese female patients with gastric cancer [33] and found a mean score \pm SD of $10.8 \pm$ 2.9. The difference between the two studies is small. However, according to the evaluation criteria [13], a score greater than 11.0 indicates a rather high tendency of repressive behavior.

Self-repression had a significant impact on psychological status. While an association between emotional repression and a depressive state has been pointed out in some studies [7] [9] [11], the impact of self-repressive traits, which include emotional and behavioral control and were commonly observed in Japanese people, on psychological vulnerability has not been examined. As defined above, the self-repressive trait was characterized as repressed true feelings or repression of one's own needs, and paying too much attention to fulfilling other people's demands or expectations [13]. Individuals with this trait are considered to be nice to the people around them [13]. For most breast cancer patients, receiving long-term treatment and playing their social role at the same time may be an extremely stressful situation. Lazarus and Folkman [27] stated when comparing major life events (i.e. diagnose of cancer or surgery) and daily hassles (i.e. far less stressful experiences in our day-to-day living), daily hassles are far superior to life events in predicting psychological and somatic symptoms. Self-repressive coping for a stressful situation may result in intensifying the stress. This accumulating stress may lead to psychological deterioration. Interestingly, physicians who were involved in this study as researchers and who 
treated patients with breast cancer on a daily basis revealed that they generally felt that patients were sociable, delightful, and expressed their opinions. The physicians seldom felt that patients were repressive or that $40 \%$ of the patients were psychologically vulnerable. However, the data and physician's impressions of the patients were inconsistent. Patients may not be expressing their true feelings or thoughts. Some researchers suggested that the psychological distress encountered by patients is likely underestimated by their nurses [34] [35]. Health care providers may need to recognize the possibility of being misled into thinking that their patients are welladjusted when, in fact, they are suffering. This information should be provided to not only to the health care providers but also to the patients. Then, health care providers need to encourage the patients to find ways to cope with their stressful situation.

In addition, perceived social support, particularly from family, was significant and the most powerful determinant of both anxiety and depression. Social support plays a role in enhancing self-esteem, buffering the effects of stress, and contributing to a better psychological status of breast cancer patients [24]-[26] [36]. In this study, social support did not play a role in decreasing the level of the self-repressive trait, thus, our hypothesis was not supported. However, regarding psychological outcomes, the study had similar findings to those of previous studies. The results suggested that lack of support from family and from significant others correlated with anxiety, and lack of support from family, friends, and significant others correlated with depression. However, among the support variables, family support had the most significant impact on depression. One study [37] suggested communication regarding cancer within the nuclear family was associated with decreased feelings of stigmatization and a reduced sense of vulnerability. Thus, health care providers should carefully monitor patients' social resources.

On the other hand, the family caregivers of breast cancer patients also experience a difficult time. Their psychological problems, such as anxiety, depression, and low overall QOL, were reported in some studies [37] [38]. The studies suggested family caregivers have information needs, and they require communication with health care providers [37]. Careful assessment of the patient and their family's needs is required. Nurses need to encourage and provide support for not only the patients but also the family members so that family members can effectively care for the patients' needs.

\section{Limitation of the Study}

There are some limitations to this study.

First, the study design is cross-sectional. These findings showed only relationships among variables and did not show the causal relationships. A longitudinal study will be needed to confirm the causal relationship among variables.

Secondly, there may be bias caused by sampling. We used nonrandom sampling, and the sample size was small. Subjects were limited to only primary breast cancer patients, and patients with recurrent disease were not included. The findings are insufficient to consider further support for any disease stage of breast cancer patients.

Thirdly, Cronbach's alpha of both the self-repression scale and the anxiety scale (anxiety scale was a subscale of HADS) were 0.67 which indicated rather low reliability. It is probable that this may affect the reliability of the study.

Finally, the study method to collect the data may be subject to bias. Patients were informed that their medical records would be referred. Even though they were informed it would not be used for matching responses in the questionnaire, there was a possibility that this information affected their responses if the patients thought that individuals could be identified. That is, patients might provide socially desirable responses, resulting in higher/ lower scores in any of the study tools. In addition, it is generally acknowledged that people who return the questionnaires tend to be cooperative. Considering the characteristics of the self-repressive trait, it is probable that patients who were more repressive tended to return the questionnaire. If this is true, the data may have been extracted from a group containing a higher proportion of repressors, though there was a variation in self- repression score (Mean \pm SD: $11.3 \pm 2.9$; actual range 3 - 18; possible range: 0 - 20).

Considering these limitations, the findings may be biased and may be difficult to generalize in other patient populations.

The variables in this study accounted for only $24 \%$ variance for anxiety and $27 \%$ variance for depression, suggesting that factors outside the scope of this study may have a greater impact on both anxiety and depression. These results indicate that further research is required to identify other potential risk factors and that these find- 
ings should be interpreted carefully.

\section{Conclusions}

We examined the psychological status and its determinants, especially focusing on the self-repressive trait in ambulatory Japanese breast cancer patients. Around $40 \%$ of the subjects scored higher than the cut-off point for anxiety and depression which indicated that they were psychologically vulnerable and may require support.

In the conceptual framework (Figure 1), self-repression and support from family were significant predictors of anxiety and depression while attributes and disease-related variables were not. Nurses need to pay more attention to patients' personal and psychosocial status and to carefully identify their needs. The information regarding the findings should be conveyed to the patients so they can advocate for themselves.

Family support can contribute to a better psychological status of the patients. However, family members may also have problems supporting the patients. Nurses need to encourage and to inform not only the patients but also to family members so that family members can effectively support patients.

\section{References}

[1] Ministry of Health Labour and Welfare. (2013) Vital Statistics Japan.

http://ganjoho.jp/professional/statistics/statistics.html

[2] Matsuda, A., Matsuda, T., Shibata, A., and the Japan Cancer Surveillance Research Group (2013) Cancer Incidence and Incidence Rates in Japan in 2007: A Study of 21 Population-Based Cancer Registries for the Monitoring of Cancer Incidence in Japan (MCIJ) Project. Japanese Journal of Clinical Oncology, 43, 328-336.

http://dx.doi.org/10.1093/jjco/hys233

[3] Kornblith, A.B. and Ligibel, J. (2003) Psychosocial and Sexual Functioning of Survivors of Breast Cancer. Seminars in Oncology, 30,799-813. http://dx.doi.org/10.1053/j.seminoncol.2003.08.025

[4] Kugaya, A., Akechi, T., Okuyama, T., et al. (1998) Screening for Psychological Distress in Japanese Cancer Patients. Japanese Journal of Clinical Oncology, 28, 333-338. http://dx.doi.org/10.1093/jjco/28.5.333

[5] Vahdaninia, M., Omidvari, S. and Montazeri, A. (2010) What Do Predict Anxiety and Depression in Breast Cancer Patients? A Follow-Up Study. Social Psychiatry and Psychiatric Epidemiology, 45, 355-361. http://dx.doi.org/10.1007/s00127-009-0068-7

[6] Andreu, Y., Galdón, M.J., Durá, E., et al. (2012) A Longitudinal Study of Psychosocial Distress in Breast Cancer: Prevalence and Risk Factors. Psychology Health, 27, 72-87. http://dx.doi.org/10.1080/08870446.2010.542814

[7] Akechi, T., Okuyama, T., Imoto, S., et al. (2001) Biomedical and Psychosocial Determinants of Psychiatric Morbidity among Postoperative Ambulatory Breast Cancer Patients. Breast Cancer Res Treat, 65, 195-202. http://dx.doi.org/10.1023/A:1010661530585

[8] Watson, M., Greer, S., Rowden, L., et al. (1991) Relationships between Emotional Control, Adjustment to Cancer and Depression and Anxiety in Breast Cancer Patients. Psychological Medicine, 21, 51-57. http://dx.doi.org/10.1017/S0033291700014641

[9] Iwamitsu, Y., Shimoda, K., Abe, H., et al. (2005) Anxiety, Emotional Suppression, and Psychological Distress before and after Breast Cancer Diagnosis. Psychosomatics, 46, 19-24. http://dx.doi.org/10.1176/appi.psy.46.1.19

[10] Bardwell, W.A., Natarajan, L., Dimsdale, J.E., et al. (2006) Objective Cancer-Related Variables Are Not Associated with Depressive Symptoms in Women Treated for Early-Stage Breast Cancer. Journal of Clinical Oncology, 24, 24202427. http://dx.doi.org/10.1200/JCO.2005.02.0081

[11] Nakatani, Y., Iwamitsu, Y., Kuranami, M., et al. (2012) Emotional Suppression and Psychological Responses to a Diagnosis of Breast Cancer. Shinrigaku Kenkyu, 83, 126-134. http://dx.doi.org/10.4992/jjpsy.83.126

[12] Schlatter, M.C. and Cameron, L.D. (2010) Emotional Suppression Tendencies as Predictors of Symptoms, Mood, and Coping Appraisals during AC Chemotherapy for Breast Cancer Treatment. Annals of Behavioral Medicine, 40, 15-29. http://dx.doi.org/10.1007/s12160-010-9204-6

[13] Munakata, T. (1996) Koudoukagaku kara mita kennkou to byouki [Health and Disease from the Perspective of Behavioral Science]. 2nd Edition, Medical-Friend Co, Tokyo.

[14] Munakata, T. (1993) Perceived Stressor and Coping Behavior-Self-Growth Growth from “Iiko” Behavior. Journal of Mental Health, 39, 29-40.

[15] Yoshida, M., Endo, K., Morita, M., et al. (2004) A Study on the Effects of a Support Group Program for Cancer Patients Open to Local Communities. Japanese Journal of Psychosomatic Medicine, 44, 133-140.

[16] Fukui, S., Kugaya, A., Okamura, H., et al. (2000) A Psychosocial Group Intervention for Japanese Women with Pri- 
mary Breast Cartinoma. Cancer, 89, 1026-1036.

http://dx.doi.org/10.1002/1097-0142(20000901)89:5<1026::AID-CNCR12>3.0.CO;2-5

[17] Fukui, S., et al. (2003) The Effect of a Psychosocial Group Intervention on Loneliness and Social Support for Japanese Women with Primary Breast Cancer. Oncology Nursing Forum, 30, 823-830. http://dx.doi.org/10.1188/03.ONF.823-830

[18] Spiegel, D. and Classen, C. (2000) Group Therapy for Cancer Patients: A Research-Based Handbook of Psychosocial Care. Basic Books, United States.

[19] Baucom, D.H., Porter, L.S., Kirby, J.S., et al. (2005-2006) Psychosocial Issues Confronting Young Women with Breast Cancer. Breast Disease, 23, 103-113.

[20] Wenzel, L.B., Fairclough, D.L., Brady, M.J., et al. (1999) Age-Related Differences in the Quality of Life of Breast Carcinoma Patients after Treatment. Cancer, 86, 1768-1774. http://dx.doi.org/10.1002/(SICI)1097-0142(19991101)86:9<1768::AID-CNCR19>3.0.CO;2-O

[21] Kissane, D.W., Grabsch, B., Love, A., et al. (2004) Psychiatric Disorder in Women with Early Stage and Advanced Breast Cancer: A Comparative Analysis. Australian \& New Zealand Journal of Psychiatry, 38, 320-326. http://dx.doi.org/10.1080/j.1440-1614.2004.01358.x

[22] Aapro, M. and Cull, A. (1999) Depression in Breast Cancer Patients: The Need for Treatment. Annals of Oncology, 10, 627-636. http://dx.doi.org/10.1023/A:1008328005050

[23] Cohen, L., Hack, T.F., de Moor, C., et al. (2000) The Effects of Type of Surgery and Time on Psychological Adjustment in Women after Breast Cancer Treatment. Annals of Surgical Oncology, 7, 427-434. http://dx.doi.org/10.1007/s10434-000-0427-9

[24] Kornblith, A.B., Herndon, J.E., Zuckerman, E., et al. (2001) Social Support as a Buffer to the Psychological Impact of Stressful Life Events in Women with Breast Cancer. Cancer, 91, 443-454. http://dx.doi.org/10.1002/1097-0142(20010115)91:2<443::AID-CNCR1020>3.0.CO;2-Z

[25] Helgeson, V.S. and Cohen, S. (1996) Social Support and Adjustment to Cancer: Reconciling Descriptive, Correlational, and Intervention Research. Health Psychology, 15, 135-148. http://dx.doi.org/10.1037/0278-6133.15.2.135

[26] Schroevers, M.J., Ranchor, A.V. and Sanderman, R. (2003) The Role of Social Support and Self-Esteem in the Presence and Course of Depressive Symptoms: A Comparison of Cancer Patients and Individuals from the General Population. Social Science \& Medicine, 57, 375-385. http://dx.doi.org/10.1016/S0277-9536(02)00366-0

[27] Lazarus, R. and Folkman, S. (1984) Stress, Appraisal, and Coping. Springer Publishing Co., New York.

[28] Kitamura, T. (1993) Hospital Anxiety and Depression Scale. Seishinka Shindangaku, 4, 371-372.

[29] Zimet, G.D., Dahlem, N.W., Zimet, S.G. and Farley, G.K. (1988) The Multidimensional Scale of Perceived Social Support. Journal of Personality Assessment, 52, 30-41. http://dx.doi.org/10.1207/s15327752jpa5201_2

[30] Iwasa, H., Gondo, Y., Mausi, Y., et al. (2007) Reliability and Validity of the Japanese Version of the Multidimensional Scale of Perceived Social Support among Middle-Aged and Elderly People Living in a Japanese Community. Journal of Health and Welfare Statistics, 54, 26-33.

[31] Zigmond, A.S. and Snaith, R.P. (1983) The Hospital Anxiety and Depression Scale. Acta Psychiatr Scand, 67, 361-370. http://dx.doi.org/10.1111/j.1600-0447.1983.tb09716.x

[32] Moorey, S., Greer, S., Watson, M., et al. (1991) The Factor Structure and Factor Stability of the Hospital Anxiety and Depression Scale in Patients with Cancer. British Journal of Psychiatry, 158, 255-259. http://dx.doi.org/10.1192/bjp.158.2.255

[33] Maeda, T., Onuoha, F.N. and Munakata, T. (2006) The Effect of Postoperative Symptom Experience, and Personality and Psychosocial Factors on Depression among Postgastrectomy Patients in Japan. Gastroenterology Nursing, 29, 437444. http://dx.doi.org/10.1097/00001610-200611000-00002

[34] Mårtensson, G., Carlsson, M. and Lampic, C. (2010) Are Cancer Patients Whose Problems Are Overestimated by Nurses Less Satisfied with Their Care? European Journal of Cancer Care, 19, 382-392. http://dx.doi.org/10.1111/j.1365-2354.2009.01070.x

[35] Mårtensson, G., Carlsson, M. and Lampic, C. (2008) Do Nurses and Cancer Patients Agree on Cancer Patients’ Coping Resources, Emotional Distress and Quality of Life? European Journal of Cancer Care, 17, 350-360. http://dx.doi.org/10.1111/j.1365-2354.2007.00866.x

[36] den Heijer, M., Vos, J., Seynaevem, C., Vanheusden, K., et al. (2012) The Impact of Social and Personal Resources on Psychological Distress in Women at Risk for Hereditary Breast Cancer. Psychooncology, 21, 153-160. http://dx.doi.org/10.1002/pon.1879

[37] Schmid-Büchi, S., Halfens, R.J., Dassen, T. and van den Borne, B. (2011) Psychosocial Problems and Needs of Posttreatment Patients with Breast Cancer and Their Relatives. European Journal of Oncology Nursing, 15, 260-266. 
T. Maeda et al.

http://dx.doi.org/10.1016/j.ejon.2009.11.001

[38] Heidari Gorji, M.A., Bouzar, Z., Haghshenas, M., Kasaeeyan, A.A, Sadeghi, M.R. and Ardebil, M.D. (2012) Quality of Life and Depression in Caregivers of Patients with Breast Cancer. BMC Research Notes, 20, 310. 